\title{
Profile of clinically-diagnosed dementias in a neuropsychiatric practice in Abeokuta, South-Western Nigeria
}

\author{
G Amoo', RO Akinyemi ${ }^{2,6}$, LU Onofa', JO Akinyemi ${ }^{3}$, O Baiyewu', AO Ogunlesi', A Ogunniyi ${ }^{5}$ \\ 'Psychogeriatrics Unit, Aro Neuropsychiatric Hospital, Abeokuta, Nigeria \\ 2Division of Neurology, Department of Medicine, Federal Medical Centre, Abeokuta, Nigeria \\ ${ }^{3}$ Department of Epidemiology and Biostatistics, College of Medicine, University of Ibadan, Ibadan, Nigeria \\ ${ }^{4}$ Department of Psychiatry, University College Hospital, Ibadan, Nigeria \\ ${ }^{5}$ Department of Medicine, University College Hospital, Ibadan, Nigeria \\ GInstitute for Ageing and Health, Newcastle University, Campus for Ageing and Vitality, Newcastle upon Tyne, NE4 5PL, UK
}

\begin{abstract}
Objective: Many subjects with dementia present primarily to neuropsychiatric practices because of behavioural and psychological symptoms (BPSD). This study reviewed the profile of clinically-diagnosed dementias and BPSD seen in a pioneer neuropsychiatric practice in Abeokuta, southwestern Nigeria over a ten year period (January 1998 - December 2007). Methods: A review of hospital records of all patients with diagnoses of dementia or dementing illness using the ICD-10 criteria as well as specific diagnostic criteria for different dementia phenotypes. Associated BPSD, co-morbidities and treatments were also reviewed. Results: Out of a total of 240,294 patients seen over the study period, 108 subjects met clinical diagnostic criteria for probable dementia giving a hospital frequency of 45 per 100,000. Alzheimer's disease (AD) and Vascular dementia (VaD) were the predominant phenotypes seen in 62 (57.4\%) and 18 (16.7\%) subjects respectively. Others include mixed dementia (4 cases), frontotemporal dementia (4 cases), Lewy body dementia (3 cases), alcohol-related dementia (3 cases), PD dementia (1 case) and unclassifiable (13 cases). Apathy, night time behaviour, aberrant motor behaviour, agitation and irritability were the most common BPSD features, while hypertension was the most common co-morbidity. Neuroleptics, anticholinergics and anti-hypertensives were most commonly prescribed. Anticholinesterase inhibitors were sparingly used. Conclusion: Probable AD was the most prevalent dementia phenotype seen in this practice. Increased awareness of dementia and better utilization of specific treatments are needed among psychiatrists and primary care practitioners in Nigeria.
\end{abstract}

Keywords: Dementia phenotypes; BPSD; Neuropsychiatric practice; Nigeria; Africa

Received: $20-10-2010$

Accepted: 11-01-2011

doi: http://dx.doi.org/10.4314/ajpsy.v14i5.5

\section{Introduction}

Dementia, a mental health disorder of global public health concern, is defined as a considerable decline in cognitive function that is severe enough to impair the ability to perform

Correspondence

Dr. RO Akinyemi

Division of Neurology, Department of Medicine, Federal Medical Centre

Abeokuta, Nigeria.

email: rufusakinyemi@yahoo.com personal activities of daily living. ${ }^{1}$ In the mid-80.s, dementia was thought to be rare in developing countries including Nigerial $^{1-3}$, however recent estimates in 2007 suggested that about 33 million people worldwide, $60-70 \%$ of whom resided in developing countries including Africa, suffered from dementia while the total cost of ageing-related dementias exceeded US\$ 70 billion. ${ }^{4}$ And, though the burden of dementia is currently low in sub-Saharan Africa, population ageing, lifestyle changes, increasing vascular factors, poverty, malnutrition, wars and the HIV pandemic may cause future increments. ${ }^{5}$ 
From community-based studies, the prevalence of dementia in Nigeria ranges between 2.29\% and $2.79 \%$ with Alzheimer's disease $(\mathrm{AD})$, whose prevalence ranges between $1.41 \%$ and $1.86 \%$, being the most common type. 6,7 However, in a previous retrospective study of clinicallydiagnosed dementing illnesses in Ibadan, 37 cases of dementia diagnosed according to the ICD-9 and DSM-IIIR criteria were seen over a six year period (1984 - 1989) out of a total of 57,440 cases admitted, giving an average hospital frequency of 64 cases per 100,000 admissions. Majority of the cases (48.7\%) had vascular dementia while secondary dementias (metabolic/toxic, subdural haematomas, organ failure) constituted 21.6\%. Thirteen percent of cases were mixed dementias while primary degenerative dementias constituted only 2.7\%. ${ }^{8}$ Associated morbidities included hypertension, diabetes mellitus, parkinsonism and benign prostatic hypertrophy. In a similar hospital-based study from Kinshasa, Democratic Republic of Congo, 35 cases of dementia were diagnosed over a ten year period (1990-2000) with $42.8 \%$ being vascular dementia while $22.8 \%$ were $\mathrm{AD} .^{9}$

Behavioural and psychological symptoms of dementia (BPSD) are common manifestations and may be the presenting symptom or appear in the course of dementia. They include delusions/paranoia, visual and auditory hallucinations, dysphoria, anxiety, agitation/aggression, euphoria, disinhibition, irritability, apathy, aberrant motor behaviour such as wandering, night-time behavior including sleep alterations, incontinence, depression, phobias, poor eating habits and schizophreniform/paranoid psychosis. 1012 BPSD constitute a major cause of care giver burden, they impair quality of life as well as increase direct and indirect cost of care and mortality. BPSD are often the trigger for seeking hospital care and placement of subjects in institutional care. ${ }^{10}$

Stemming from the paucity of data on dementia in psychiatry practices across black Africa, we reviewed the profile of clinically-diagnosed dementias seen in a pioneer neuropsychiatric practice in Abeokuta, Southwestern Nigeria over a ten year period. The frequency and types of dementing illnesses as well as the characteristics of BPSD, co-morbidities and treatment modalities were assessed.

\section{Methods}

We obtained data from hospital records of all patients with a provisional diagnosis of dementing illness or dementia managed at the Aro Neuropsychiatric Hospital, Abeokuta, Southwestern Nigeria, over a ten year period (January 1998 - December 2007) using a semi-structured proforma. The case records were coded according to the 10th revision of the International Classification of Diseases.

Consensus diagnosis involving a Neurologist (ROA) and two Psychiatrists (GA, LUO) was made on each of the cases using the International Classification of Diseases Tenth Revision (ICD-10) criteria for dementia. ${ }^{15}$ Severity was assessed using the Clinical Dementia Rating Scale (CDR). ${ }^{16}$ For specific dementia phenotypes, the National Institute for Neurological and Communicative Disorders and Stroke and the Alzheimer Disease and Related Disorders Association (NINCDS - ADRDA) criteria for Alzheimer's disease (AD) ${ }^{17}$, NINCDS -AIRENS criteria for Vascular dementia (VaD) ${ }^{18}$,
McKeith criteria for Dementia with Lewy bodies(DLB) ${ }^{19}$, Lund and Manchester Criteria for Frontotemporal dementia (FTD) ${ }^{20}$ and the Clinical Diagnostic criteria for Parkinson disease Dementia (PDD) ${ }^{21}$ were utilized. The ICD-10 criteria included cognitive impairment in a conscious and alert state, functional and physical impairment resulting from above, persistence of symptoms for at least 6 months, and absence of clouding of consciousness. The CDR used all available information to rate a subject's performance in each of six categories (memory, orientation, judgement, community affairs, home and hobbies and personal care) and categorizes severity into mild (0.5 - 1.0), moderate (2.0) and severe (3.0).

A comprehensive symptom check list was generated for assessing the domains represented in the Neuropsychiatric Inventory - Questionnaire (NPI-Q) ${ }^{22}$ and applied retrospectively to all the cases meeting the criteria for probable dementia. The frequency of occurrence of BPSD in the domains of the NPI-Q was noted but the retrospective nature of the study precluded the determination of the severity of the symptoms as well as distress in the caregivers/informant.

The protocol received due approval from the Health Research Committee of the hospital. Data was entered using the Epidata software, cleaned and subsequently analyzed using the Statistical Package for Social Sciences version 15.0 (SPSS Chicago Inc.). Descriptive statistics including frequency tables and cross tabulations of sociodemographic and clinical variables were undertaken. Continuous data were expressed as means (standard deviation) (SD), median and range. Categorical variables were compared using the chi square test while group differences of continuous variables were compared using the student's t-test. All statistical tests were carried out at 5\% significance level.

\section{Results}

\section{Hospital frequency of dementia}

Over the 10-year period, a total of 240,294 patients presented to the hospital, of which 125 received a diagnosis of dementing illness. However, only 108 subjects met the ICD-10 criteria for clinical diagnosis of probable dementia, giving an average hospital frequency of 45 cases per 100,000 patients. Seventeen patients were excluded and their diagnoses were: depressive illness (4), delirium (2), schizophrenia (2) and unclassified psychotic disorder (9).

The 108 patients diagnosed with dementia consisted of $51(47.2 \%)$ males and 57 (52.8\%) females, age [mean (SD) 70.1 (9.8) years, median 70, range 44 to 101]. Ten patients were younger than 60 years of age while 43 (39.8\%) were in the 60 -69 years age range. Majority were married (63.5\%), had no formal education (63.0\%), were of Yoruba ethnic extraction (96.3\%), were retired (98.1\%), lived with others (93.5\%) and were seen on an outpatient basis (86.1\%).

\section{Dementia phenotypes}

Table I shows the distribution, demographic and clinical features of the different dementia phenotypes seen among the subjects. AD predominated with 62 cases (57.4\%) while 


\begin{tabular}{|c|c|c|c|c|c|c|c|c|}
\hline Variable & $A D(n=62)$ & $\operatorname{VaD}(n=18)$ & Mixed $D(n=4)$ & $F T D(n=4)$ & $D L B(n=3)$ & $A / c D(n=3)$ & $P D D(n=1)$ & $\begin{array}{l}\text { Unclassified } \\
(n=13)\end{array}$ \\
\hline $\begin{array}{l}\text { Mean age ( SD) years } \\
\text { Median duration of symptoms (range) mths }\end{array}$ & $\begin{array}{l}72.8(9.4) \\
14(4-99)\end{array}$ & $\begin{array}{l}64.5(8.7) \\
23(3-78)\end{array}$ & $\begin{array}{l}66.3(5.4) \\
16(3-57)\end{array}$ & $\begin{array}{l}69.3(17.1) \\
64(39-73)\end{array}$ & $\begin{array}{l}65.0(5.0) \\
13(7-15)\end{array}$ & $\begin{array}{l}66.3(14.6) \\
37(7-37)\end{array}$ & $\begin{array}{l}70.0(0) \\
6(0)\end{array}$ & $\begin{array}{l}67.4(9.1) \\
12(4-101)\end{array}$ \\
\hline $\begin{array}{l}\text { Gender: } \mathrm{n}(\%) \\
\text { Male } \\
\text { Female }\end{array}$ & $\begin{array}{l}24(38.7) \\
38(61.3)\end{array}$ & $\begin{array}{l}13(72.2) \\
5(27.8)\end{array}$ & $\begin{array}{l}2(50.0) \\
2(50.0)\end{array}$ & $\begin{array}{l}2(50.0) \\
2(50.0)\end{array}$ & $3(100.0)$ & $3(100.0)$ & $1(100.0)$ & $\begin{array}{l}6(46.2) \\
7(53.8)\end{array}$ \\
\hline $\begin{array}{l}\text { Level of Education: } \mathrm{n}(\%) \\
\text { None } \\
\text { At least primary }\end{array}$ & $\begin{array}{l}46(67.6) \\
16(32.4)\end{array}$ & $\begin{array}{l}9(50.0) \\
9(50.0)\end{array}$ & $\begin{array}{l}2(50.0) \\
2(50.0)\end{array}$ & $\begin{array}{l}1(25.0) \\
3(75.0)\end{array}$ & $\begin{array}{l}3(100.0) \\
-\end{array}$ & $3(100.0)$ & $1(100.0)$ & $\begin{array}{l}6(46.2) \\
7(53.8)\end{array}$ \\
\hline $\begin{array}{l}\text { Clinical Dementia Rating (CDR) } \\
\text { Mild }(0.5-1) \\
\text { Moderate (2.0) } \\
\text { Severe (3.0) }\end{array}$ & $\begin{array}{l}5(8.3) \\
28(46.7) \\
27(45.0)\end{array}$ & $\begin{array}{l}1(5.6) \\
5(27.8) \\
12(66.7)\end{array}$ & $\begin{array}{l}- \\
1(25.0) \\
3(75.0)\end{array}$ & $\begin{array}{l}- \\
3(75.0) \\
1(25.0)\end{array}$ & $\begin{array}{l}2(66.7) \\
1(33.3)\end{array}$ & $\begin{array}{l}- \\
1(33.3) \\
2(66.7)\end{array}$ & 1(100.0) & $\begin{array}{l}2(15.4) \\
6(46.2) \\
5(38.4)\end{array}$ \\
\hline $\begin{array}{l}\text { Cognitive symptoms [n (\%)] } \\
\text { Impairment of recent memory } \\
\text { Language problems } \\
\text { Visuospatial dysfunction } \\
\text { Attentional deficits } \\
\text { Disorientation in time } \\
\text { Difficulty recognizing faces }\end{array}$ & $\begin{array}{l}58(93.5) \\
54(87.1) \\
48(77.4) \\
49(79.0) \\
47(75.8) \\
55(88.7)\end{array}$ & $\begin{array}{l}14(77.8) \\
14(77.8) \\
7(38.9) \\
12(66.7) \\
13(72.2) \\
11(61.1)\end{array}$ & $\begin{array}{ll}4 & (100.0) \\
2 & (50.0) \\
2 & (50.0) \\
3 & (75.0) \\
2 & (50.0) \\
2 & (50.0)\end{array}$ & $\begin{array}{l}4(100.0) \\
3(100.0) \\
1(25.0) \\
3(75.0) \\
4(100.0) \\
3(75.0)\end{array}$ & $\begin{array}{l}3(100.0) \\
3(100.0) \\
1(33.3) \\
3(100.0) \\
3(100.0) \\
3(100.0)\end{array}$ & $\begin{array}{l}3(100.0) \\
3(100.0) \\
1(33.3) \\
2(66.7) \\
2(66.7) \\
3(100.0)\end{array}$ & $\begin{array}{l}1(100.0) \\
1(100.0) \\
- \\
1(100.0) \\
1(100.0) \\
1(100.0)\end{array}$ & $\begin{array}{l}13(100.0) \\
8(61.5) \\
7(53.8) \\
10(76.9) \\
9(69.2) \\
8(61.5)\end{array}$ \\
\hline $\begin{array}{l}\text { Medical Co-morbidities } \\
\text { Hypertension [BP >140/90mmHg] n (\%) } \\
\text { Stroke } \\
\text { Diabetes Mellitus } \\
\text { Osteoarthritis } \\
\text { Parkinson's disease }\end{array}$ & $\begin{array}{l}17(27.4) \\
- \\
3(4.8) \\
5(8.1) \\
-\end{array}$ & $\begin{array}{l}17(94.4) \\
13(72.2) \\
2(11.1) \\
1(5.6) \\
-\end{array}$ & $\begin{array}{l}3(75.0) \\
- \\
- \\
-\end{array}$ & $\begin{array}{l}- \\
- \\
- \\
-\end{array}$ & $\begin{array}{l}1(33.3) \\
- \\
- \\
- \\
-\end{array}$ & $\begin{array}{l}1(33.3) \\
- \\
1(33.3) \\
- \\
-\end{array}$ & $\begin{array}{l}- \\
- \\
- \\
- \\
1(100.0)\end{array}$ & $\begin{array}{l}- \\
- \\
- \\
-\end{array}$ \\
\hline
\end{tabular}

VaD was diagnosed in 18 cases (16.7\%). Others included mixed dementia (4 cases [3.7\%]) FTD (4 cases [3.7\%]), DLB (3 cases [2.8\%]), alcohol-related dementia (3 cases [2.8\%]), and PDD (1 case [0.9\%]). In 13 cases (12.0\%), lack of relevant neuroimaging, biochemical and haematological investigations precluded the determination of more specific dementia diagnoses. Mild, moderate and severe dementia were present in 10 (9.3\%), 47 (43.5\%) and 51 (47.2\%) of the subjects respectively. The CDR ratings according to phenotypes are also summarized in the second panel of Table I.

\section{Clinical characteristics of the dementia phenotypes}

The mean age of onset among subjects with $\mathrm{AD}$ was $71.2 \pm$ 9.4 years and majority of subjects (44/62) were older than 70 years $\left(\mathrm{x}^{2}=19.5, \mathrm{p}=0.001\right)$. There was a statistically significant female preponderance [male to female ratio 0.63:1, $\mathrm{x}^{2}=4.23, \mathrm{p}=0.04$ ] as well as a significant preponderance of persons with no formal education $\left(\mathrm{x}^{2}=\right.$ 8.88, $\mathrm{p}=0.031$ ). Subjects with AD were significantly older than subjects with other dementia subtypes $(T=3.61, P$ $<0.001$ ). Among subjects with VaD, mean age of onset was $62.8 \pm 8.7$ years with a significantly higher male proportion [male to female ratio 2.6:1, $\mathrm{x}^{2}=5.41, \mathrm{p}=0.02$ ]. The mean age of onset for subjects with mixed dementia was $64.6 \pm$ 5.4 years with equal gender balance. The mean ages of onset for other phenotypes were: FTD $(65 \pm 17.1$ years $)$; alcohol- related dementia (64.9 \pm 14.6 years); PDD (69.5 years) and DLB (64. $1 \pm 5.0$ years).

Impairment of recent memory was the most common cognitive symptom recorded across the different phenotypes. Table I shows the spectrum of other cognitive symptoms.

Hypertension (BP > 140/90mmHg) was the most common co-morbidity and was seen in

$17 / 62$ (27.4\%), 17/18 (94.4\%) and 3/4 (75\%) of subjects with $\mathrm{AD}, \mathrm{VaD}$ and mixed dementia respectively. Previous stroke 13/18 (72.2\%), post-stroke parkinsonism (1/18) and post-stroke seizure disorder (1/18) were recorded mainly in the VaD subgroup while Parkinson's disease was diagnosed in the only case with PDD. Hearing impairment was documented in four subjects with probable $\mathrm{AD}$, while visual impairment was documented in a case each of $\mathrm{AD}$ and $\mathrm{VaD}$. Computer tomography scan in three subjects with $\mathrm{AD}$ revealed gross cerebral atrophy with ventricular enlargement. 
Table II: Profile of BPSD and Dementia phenotype based on the Neuropsychiatry Inventory-Questionnaire (NPI -Q) schema

\begin{tabular}{|c|c|c|c|c|c|c|c|c|c|}
\hline$B P S D$ & $\begin{array}{l}\text { +Baiyewu } \\
\text { et al }(n=40)\end{array}$ & $\begin{array}{l}A D \\
(n=62)\end{array}$ & $\begin{array}{l}\mathrm{VaD} \\
(n=18)\end{array}$ & $\begin{array}{l}\text { Mixed D } \\
(n=4)\end{array}$ & $\begin{array}{l}\text { FTD } \\
(n=4)\end{array}$ & $\begin{array}{l}D L B \\
(n=3)\end{array}$ & $\begin{array}{l}A / c D \\
(n=3)\end{array}$ & $\begin{array}{l}P D D \\
(n=1)\end{array}$ & $p$-value \\
\hline $\begin{array}{l}\text { Delusions /Paranoia } \\
\text { Hallucinations } \\
\text { Dysphoria/Depression } \\
\text { Anxiety } \\
\text { Agitation/Aggression } \\
\text { Euphoria } \\
\text { Disinhibition } \\
\text { Irritability } \\
\text { Apathy } \\
\text { Aberrant motor behaviour } \\
\text { Night-time behaviour }\end{array}$ & $\begin{array}{l}7(17.5) \\
5(12.5) \\
13(32.5) \\
8(20.0) \\
8(20.0) \\
4(10.0) \\
4(10.0) \\
11(27.5) \\
10(25.0) \\
3(7.5) \\
7(17.5)\end{array}$ & $\begin{array}{l}22(35.5) \\
16(25.8) \\
16(25.8) \\
4(6.5) \\
38(61.3) \\
3(4.8) \\
24(38.7) \\
32(51.6) \\
54(87.1) \\
48(77.4) \\
50(80.6)\end{array}$ & $\begin{array}{l}2(11.1) \\
2(11.1) \\
2(11.1) \\
2(11.1) \\
4(22.2) \\
1(5.6) \\
10(55.6) \\
4(22.2) \\
14(77.8) \\
7(38.9) \\
10(55.6)\end{array}$ & $\begin{array}{l}1(25.0) \\
1(25.0) \\
2(50.0) \\
1(25.0) \\
2(50.0) \\
- \\
3(75.0) \\
2(50.0) \\
3(75.0) \\
2(50.0) \\
2(50.0)\end{array}$ & $\begin{array}{l}- \\
2(50.0) \\
1(25.0) \\
- \\
2(50.0) \\
- \\
- \\
2(50.0) \\
2(50.0) \\
1(25.0) \\
4(100.0)\end{array}$ & $\begin{array}{ll}1 & (33.3) \\
3 & (100.0) \\
- & \\
- \\
1 & (33.3) \\
1 & (33.3) \\
- \\
1 & (33.3) \\
3 & (100.0) \\
1 & (33.3) \\
3 & (100.0)\end{array}$ & $\begin{array}{l}1(33.3) \\
- \\
- \\
- \\
2(66.7) \\
- \\
1(33.3) \\
2(66.7) \\
1(33.3) \\
1(33.3) \\
1(33.3)\end{array}$ & $\begin{array}{l}- \\
1(100.0) \\
2(100.0) \\
- \\
- \\
- \\
- \\
- \\
1(100.0) \\
- \\
-\end{array}$ & $\begin{array}{l}0.197 \\
0.244 \\
0.042^{\star} \\
0.488 \\
0.610 \\
0.385 \\
0.000^{\star} \\
0.733 \\
0.024^{\star} \\
0.016^{\star} \\
0.002^{\star}\end{array}$ \\
\hline
\end{tabular}

+Baiyewu et al (2003) studied 40 community-dwelling subjects with dementia (AD = 39; 1 = non-specific dementia) [13]

$x$ chi-square test of association between BPSD and severity of dementia on the Clinical Dementia Rating (CDR) scale.

*Value of $\mathrm{p}<0.05$ (statistically significant).

\section{Spectrum of behavioural and psychological symptoms (BPSD)}

Table II shows the spectrum of BPSD documented among subjects with the various dementia phenotypes using the Neuropsychiatric Inventory (NPI-Q) schema in comparison with the profile obtained by Baiyewu et al among 40 communitydwelling residents with dementia (largely AD). ${ }^{13}$ Apathy was the most common BPSD across all the phenotypes, documented in $87.1 \%, 77.8 \%$ and $75 \%$ of subjects with $\mathrm{AD}, \mathrm{VaD}$ and mixed dementia respectively. Hallucinations (visual) were documented in all the cases diagnosed of DLB and PDD. Generally, the frequencies of the various BPSDs tended to be higher in the hospital cohort than the community-dwelling cohort studied by Baiyewu et al, most especially agitation, disinhibition, irritability, apathy, aberrant motor behaviour (wandering) and night time behavior (poor sleep). There was a statistically significant association between the degree of dementia severity and disinhibition $(p<0.001)$, apathy $(p=0.024)$, aberrant motor behaviour ( $p=0.016)$, dysphoria/depression $(p=0.042)$ and night time behaviour $(\mathrm{p}=0.002)$.

\section{Treatments}

The majority of the patients, 104 (96.3\%), received antipsychotic medications (chlorpromazine, trifluoperazine, thioridazine, risperidone, haloperidol, flupenthixol, fluphenazine decanoate) while 36 (33.3\%) and 13 (12.0\%) received anticholinergic (benzhexol, biperiden) and antidepressant (amitriptyline, imipramine, paroxetine, meclobemide, sertraline) drugs respectively. Only l subject received an anticholinesterase inhibitor (donepezil). Fortytwo (38.9\%) and 12 (11.1\%) subjects received antihypertensive (amlodipine, nifedipine, alpha methyl dopa, amiloride + hydrochlorothiazide (moduretic), reserpine + clopamide + dihydroergocristine (brinerdin) and antidiabetic (glibenclamide, metformin and insulin) agents respectively.

\section{Discussion}

This study revealed a rather low hospital frequency of dementia. While this may be a reflection of the current low prevalence of dementia among the Nigerian elderly population ${ }^{6,7}$, it may also be due to low case ascertainment and the culture of regarding early features of dementia as part of normal ageing ${ }^{12}$ with hospital presentation becoming necessary only when moderate to severe BPSD set in, and overwhelm the relatives and caregivers. ${ }^{10-12}$

The predominant dementia phenotype was AD followed by $\mathrm{VaD}$ unlike findings from similar retrospective hospitalbased studies from Ibadan and Kinshasa respectively ${ }^{8,9}$ which reported preponderance of $\mathrm{VaD}$. We attribute this to the treatment seeking behaviour of subjects with VaD presenting to physicians/neurologists in conventional hospitals (such as those involved in the Ibadan and Kinshasa studies) on account of variable neurologic deficits associated with vascular events even before the onset of cognitive symptoms. On the other hand, patients with degenerative dementias such as AD (especially moderate to severe cases) might have presented to the neuropsychiatric hospital on account of significant BPSD that had become distressing for the family and caregivers to cope with.

The relationship between age and gender, and $\mathrm{AD}$ and $\mathrm{VaD}$ in this study is in accordance with previous reports. ${ }^{1-3,5-9}$ However, whereas previous studies from Nigeria found no significant relationship between education and $\mathrm{AD}^{6,7}$, this current study found a positive association between $\mathrm{AD}$ and lack of formal education. This is in accordance with the protective role of education against dementia by enhancing cognitive reserve. ${ }^{36}$

Dementia with Lewy bodies and FTD have been reported among Nigerians s $^{23,24}$ and prominent BPSD similarly might have accounted for the cases seen in this study. Alcohol use is quite low in Nigeria largely because of religious reasons ${ }^{25}$ and most patients with Parkinson's disease are seen in neurology practices. These may account for the relatively low frequencies of dementias related to alcohol and PD in this cohort.

Memory symptoms are often regarded as part of normal ageing in many African cultures and medical attention is sought only when BPSD become worrisome. ${ }^{12,26}$ This might explain the long duration of symptoms before presentation in hospital in 
search of treatment as well as the fact that majority of the patients were already in moderate to severe stages of the disease before medical attention was sought.

The prevalence of BPSD was quite high in this cohort, and frequencies were higher than those reported earlier by Baiyewu et al in a cohort of community-dwelling dementia subjects (predominantly $\mathrm{AD}$ ) of similar ethnic and cultural background ${ }^{13}$ but similar to high rates reported in some western outpatient dementia clinics and nursing homes. ${ }^{37}$ Apathy, night time behavior (poor sleep), aberrant motor behaviour (wandering), depression and disinhibition - all potentially distressing and embarrassing behaviours - were the BPSD most associated with the severity of the disease in our subjects. This agreed with the findings of Baiyewu et al (2003) who also observed BPSD being associated more with moderate-severe distress among caregivers of the community-dwelling cohort. ${ }^{13}$ This could, therefore, explain why subjects in the hospital cohort were brought to the neuropsychiatric hospital by their caregivers/relatives in the first place.

In the Yoruba culture, elderly subjects usually live in multigenerational extended family systems where they receive a lot of stimulation and care from the younger family members. ${ }^{27,28}$ In this study, about $95 \%$ of our subjects lived with others, especially their children.

A relatively high proportion of our subjects were hypertensive (94.4\% and $27.4 \%$ respectively among subjects with $\mathrm{VaD}$ and $\mathrm{AD}$ ) while 1 1.1\% were diabetic. Hypertension and other vascular factors are important in the aetiopathogenesis of both $\mathrm{AD}$ and $\mathrm{VaD}$. Populations with higher burden of vascular risk factors have been shown to have higher burden of both $\mathrm{AD}$ and $\mathrm{VaD}$ compared to populations of similar genetic stock but with lower burden of vascular risk factors. ${ }^{6,29}$ Significant vascular pathologies including haemorrhages, infarcts, small vessel pathologies and white matter changes have been documented in brain tissues of subjects with $\mathrm{VaD}$ and $\mathrm{AD}{ }^{30,31}$ Studies have also demonstrated increased deposition and decreased clearance of amyloid $\beta$, microangiopathy, neuronal loss and macrovascular cerebral disease in association with DM. ${ }^{32}$ Evidence similarly exists showing that effective control of vascular risk factors especially from mid-life results in significant reduction in the burden of $\mathrm{AD}$ and $\mathrm{VaD} .{ }^{33}$

Typical antipsychotic medications for BPSD constituted the majority of medications prescribed for subjects in our study cohort. Although a recent guideline ${ }^{34}$ advocates the use of an atypical antipsychotic agent, quetiapine, in the management of $\mathrm{BPSD}$, it is very expensive and largely unavailable and unaffordable in low resource settings including Nigeria. And, even though $\mathrm{AD}$ was the predominant dementia phenotype seen in this study, only one patient received donepezil. We attribute this to several factors including: problems of availability, accessibility, affordability as well as possible poor awareness of dementia management among the treatment providers during the period covered by the study. Dementia-specific continuous medical educational programmes are needed among GPS, Internists and Psychiatrists to enhance their knowledge, confidence and skills in the detection and management of dementia. It is also important to make specific dementia medications available and affordable in developing countries which are likely to bear the lion share of future increments in the global burden of dementia. ${ }^{35}$

Although the majority (>95\%) of patients in this study lived with relations, especially children, current trends show that the traditional multi-generational extended family system is already being eroded as a result of increasing urbanization and globalization..$^{27,28}$ It is therefore pertinent to evolve policies and strategies to care for the increasing population of the elderly, especially, in the developing economies of Africa. New approaches to the delivery of basic, accessible, affordable and appropriate geriatric health care are needed.

This study represents the first attempt at reviewing the profile of dementias in a neuropsychiatric hospital practice across subSaharan Africa using standardized clinical diagnostic criteria, and it shows that subjects with dementia (predominantly $\mathrm{AD}$ and $\mathrm{VaD}$ ) are seen; BPSDs are more common than in populationbased cohorts; hypertension is the most significant medical co-morbidity and significant dementia -specific treatment gap exists.

The study, however, has limitations. Its retrospective design did not allow for more rigorous and exhaustive diagnostic procedures in the subjects diagnosed with dementia. Also, none of the diagnoses was confirmed at autopsy and objective cognitive assessment tools were not routinely used in assessing the patient population. Further prospective, multi-centre studies are needed as well as specific manpower development in Psychogeriatrics in order to enhance early detection and appropriate management of dementias in general medical and psychiatric practices across Africa.

\section{Conclusion}

Our study provides data on the profile of dementias as seen in a black African neuropsychiatric practice and demonstrates that $\mathrm{AD}$ and $\mathrm{VaD}$ are the most common phenotypes similar to findings from population-based studies from developing and developed regions. The burden of BPSD appears to be higher in hospital-based than community-based cohorts. Hypertension is an important co-morbidity and significant treatment gap exists in dementia phenotype-specific treatments.

\section{References}

1. Ogunniyi A, Akinyemi RO. Dementia in Africa - a review. Archives of Ibadan Medicine 2010; 10 (1\&2): 40 -46.

2. Osuntokun BO, Ogunniyi A, Junaid TA, Lekwauwa UG, Adeuja AOG, Bademosi O. Epidemiology of Dementia in Nigeria. In Chopra.J.S, Jagannathan K, Sawhney I.M.S, (eds) Advances in Neurology. Proceedings of the XIVth World Congress of Neurology October 2027, 1989. New Delhi, India.331-342. Amsterdam. Excerpta Medica, 1990.

3. Osuntokun BO, Ogunniyi A. Epidemiology of Neurologic illness in Africa. Wemilore Press ; 1994: 36-39.

4. The Lancet Neurology Editorial. Time to confront the global dementia crisis. Lancet Neurol 2008; 7: 761.

5. Kalaria RN, Maestre GE, Arizaga R, Friedland RP, Galasko D, Hall K et al. Alzheimer's disease and vascular dementia in developing countries: prevalence, management and risk factors. Lancet Neurol 2008; 7: $812-826$.

6. Ogunniyi A, Baiyewu O, Gureje O, Hall KS, et al. Epidemiology of dementia in Nigeria: results from the Indianapolis-Ibadan study. Euro. J. Neurol 2000; 7: 485-490.

7. Yusuf AJ, Baiyewu O, Sheikh TL, Shehu AU. Prevalence of dementia and dementia subtypes among community-dwelling elderly people in northern Nigeria. Int Psychoger 2010;18:1-8.

8. Ogunniyi AO, Lekwauwa UG, Falope ZF, Osuntokun BO. Clinically 
diagnosed dementing illness in Ibadan. Afr J Med Sci 1993; 22: 61-64.

9. Kayembe TK, Ngeleka CM, Kashama JM. Etiologic and Clinical Profiles of Dementia in Kinshasa. In Proceedings of the WHO-WFNIBRO Sponsored Meeting on "Brain Ageing and Dementia in Developing Countries" April 10-13, 2007.

10. Hersch EC, Falzgraf S. Management of the behavioural and psychological symptoms of dementia. Clinical Interventions in Aging 2007; 2 (4): $611-621$

11. Hwang J-P, Yang C-H, Tsai S-J, Liu K-M. Behavioural disturbances in psychiatric inpatients with dementia of the Alzheimer type in Taiwan. Int J Geriatr. Psychiatr 1997; 12: 902 - 906.

12. Ogunniyi A, Hall KS, Baiyewu O, Gureje O, Hendrie HC Culture and Dementia. Brain Aging 2002; 2 (3): 23 -29.

13. Baiyewu O, Smith-Gamble V, Akinbiyi A, Lane KA, Hall KS, Ogunniyi $A$, et al. Behavioural and caregiver reaction of dementia as measured by the neuropsychiatric inventory in Nigerian community residents. Int Psychogeriatr 2003; 15 (4): 399 -409.

14. Jelic $V$, Winblad B. Treatment strategies in Alzheimer's disease. Brain Aging. 2002; 2 (3): 3 -8.

15. World Health Organization: Manual for International classification of diseases, injuries and causes of death. 10th Revision. WHO: Geneva 1994.

16. Hughes CP, Berg L, Danziger WL, Coben LA, Martin RL. A new clinical scale for the staging of dementia. Br J Psychiatry 1982; 140: $566-572$

17. McKhann G, Drachman D, Folstein M, Katzman R, Price D, Stadlan EM. Clinical diagnosis of Alzheimer's disease: report of the NINCDS - ADRDA Work Group under the auspices of Department of Health and Human Services Task Force on Alzheimer's disease. Neurology 1984; 34: $939-944$

18. Roman GC, Tatemichi TK, Erkinjuntti T, et al. Vascular dementia : diagnostic criteria for research studies. Report of the NINDS - AIREN International Workshop. Neurology 1993; 43: 250 -260.

19. McKeith IG, Galasko D, Kosaka K, et al. Consensus guidelines for the clinical and pathological diagnosis of dementia with Lewy bodies (DLB): report of the consortium on DLB international workshop. Neurology 1993; 47: 1113-1124.

20. Neary D, Snowden JS, Gustafson L, Passant U, Stuss D, Black S, et al. Frontotemporal lobar degeneration: a consensus on clinical diagnostic criteria. Neurology 1998; 51: 1546-1552.

21. Dubois B, Burn D, Goetz C, Aarsland D, Brown RG, Broe GA, et al. Diagnostic procedures for Parkinson's disease dementia: recommendations from the movement disorder society task force. Mov Disord 2007; 22 (16): 2314 -2324

22. Cummings JL, Mega M, Gray K, Rosemberg -Thompson S, Carusi DA, et al. The Neuropsychiatric Inventory: Comprehensive assessment of psychopathology in dementia. Neurology 1994; 44: 2308 - 2314
23. Ogunniyi A, Akang EE, Gureje O, Takao M, Piccardo P, Baiyewu O, et al. Dementia with Lewy bodies in a Nigerian: a case report. Int Psychogeriatr 2002; 14 (2): 211 -218.

24. Akinyemi RO, Owolabi MO, Makanjuola VA, Ogunseyinde AO, Ogunniyi A. Frontotemporal dementia in a Nigerian woman: case report and brief review of the literature. Afr J Med Med Sci 2009; 38: $71-75$.

25. Ohaeri JU, Odejide OA. Admissions for drug and alcohol-related problems in Nigerian psychiatric care facilities in one year. Drug Alcohol Depend 1993 Jan;31 (2):101-109.

26. Ihezue UH, Okpara E. Psychiatric disorders of old age in Enugu, Nigeria. Acta Psychr Scand1 989;79: 332 -337.

27. Akanji BO, Ogunniyi A, Baiyewu O. Healthcare for Older Persons, $A$ country Profile: Nigeria. Journal Amer. Geriatr Society 2002; 50 (7): $1289-1292$

28. Ogunniyi A, Hall KS, Baiyewu O, Gureje O, Unverzagt FW, Gao S, Hendrie HC. Caring for individuals with dementia: the Nigerian experience. West Afr J Med 2005; 24 (3): 259 -262.

29. Hendrie HC, Ogunniyi A, Hall KS, Incidence of dementia and Alzheimer's disease in two communities: Yoruba residing in Ibadan, Nigeria and African Americans residing in Indianapolis, Indiana. JAMA 2001; 285: 739 -747.

30. Kalaria RN, Erkinjuntti T. Small vessel diseases of the brain. In: LarsOlof Wahlund, Timo Erkinjuntti, Serge Gauthier (eds) Vascular cognitive impairment in Clinical Practice. Cambridge. Cambridge University Press. 2009; $118-130$.

31. Breteler MMB. Vascular risk factors for Alzheimer's disease: an epidemiological perspective. Neurobiol of Aging 2000; 21: 153 160.

32. Roberts RO, Geda YE, Knopman DS, Christianson TJH, Pankratz VS, Boeve BF et al. Association of Duration and Severity of Diabetes Mellitus with Mild Cognitive Impairment. Arch Neurol 2008; 65 (8): $1066-1073$

33. Duron E, Hanon O. Vascular risk factors, cognitive decline and dementia. Vasc Health Risk Manag 2008; 4(2):363-81.

34. Skoog I, Lithell H, Hansson L, et al. SCOPE Study Group. Effect of baseline cognitive function and antihypertensive treatment on cognitive and cardiovascular outcomes: Study on COgnition and Prognosis in the Elderly (SCOPE). Am J Hypertens 2005; 18:1052-9.

35. Ferri CP, Prince $M$, Brayne $C$, et al. Global prevalence of dementia: a Delphi consensus study. Lancet 2005; 366: 2112 -2117.

36. Ngandu T, von Strauss E, Helkala EL, Winblad B, Nissinen A, Tuomilehto J, et al. Education and dementia: what lies behind the association? Neurology 2007 Oct 2;69 (14):1442-50.

37. Zaudig M. A risk-benefit assessment of risperidone for the treatment of behavioural and psychological symptoms in dementia. Drug Safety 2000; 23: 183 -95. 